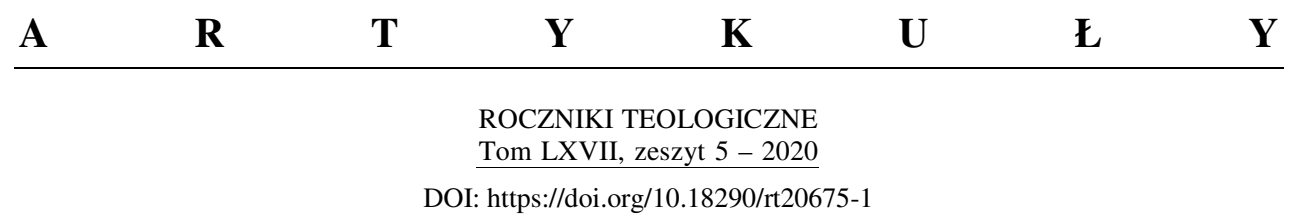

RÉMY KUROWSKI SAC

\title{
VIRTUALITÉ ET SACRAMENTALITÉ DANS LA VIE SPIRITUELLE
}

\author{
THE EFFECT OF VIRTUAL REALITY ON SACRAMENTAL REALITY \\ AND SPIRITUAL LIFE
}

\begin{abstract}
The ongoing pandemic is increasing the use of remote means of communication. Online ministry, already very present in the pastoral fabric of the Church, is growing. This continues to raise questions for Christian theology. We can use the Covid-19 experience in the Catholic Community in Hong Kong to raise questions and fuel new thinking for pastoral ministry. Ministering online raises the question of effectiveness, necessitates prioritizing community-centric actions, obliges us to reconsider intra-ecclesiastical relations, and exposes latent individualism and the fragility of the sacramental bond of belonging to the Body of Christ that is the Church. It is also instructive with regard to instincts for spiritual survival on the one hand, and vulnerability on the other. To make room for such novelty, focusing on the ongoing conversion of hearts is key, together with seeking the theological value of real communion conveyed by the virtual Eucharist Mass.
\end{abstract}

Key words: communication; communion; real and online ministry; intra-ecclesiastical relations; sacramentality; spirituality; pastoral action.

\section{INTRODUCTION}

Depuis son apparition, la virtualité comme moyen technique de communication à distance questionne la théologie chrétienne. Elle touche directement à la vie de l'Eglise et sa raison d'être ${ }^{1}$. La virtualité n'est pas seulement pour

Dr RÉMY KUROwSKI SAC - docteur en Théologie dogmatique (spécialité ecclésiologie) Institut Catholique de Paris (1993); docteur en Histoire moderne (spécialité histoire des mentalités) Sorbonne Paris IV (1993); affiliation: Institut Catholique de Paris; adresse: 16, Caine road 1509, Central, RAS, Hong Kong, remykurowski@gmail.com; ORCID: https://orcid.org/0000-0002-4657-9367.

${ }^{1}$ Rémy KuROwsKI, “Lieu d'Église virtuel”, in: Cahier de l'Atelier $\mathrm{n}^{\circ}$ 549, avril-juin 2011, et La Documentation catholique $\mathrm{n}^{\circ}$ 2475, 2 octobre 2011, p. 834-838, https://www.la-croix.com/Urbi-et-Orbi/ Archives/Documentation-catholique-n-2475/Internet-lieu-d-Eglise-virtuel 
l'Eglise le moyen d'être missionnaire. Elle est présente dans ses structures internes comme un maillage au service de la vie pastorale. L'accompagnement spirituel qui s'y effectue doit aussi le prendre en compte. La virtualité force à reconnaître sa place dans le dispositif pastoral.

Dans la période de la pandémie en cours, le recours accru à ce type de moyens de communication à distance constitue un facteur accélérant la prise de conscience de la nécessité de s'y positionner pastoralement, mais de façon théologiquement fondée. L'urgence se fait sentir au risque de ne pas pouvoir "correctement" rattraper le retard dans l'accompagnement de ce phénomène à l'intensité nouvelle, jamais égalée. Et si on n'y prend pas garde, ce sera d'autant plus difficile que les habitudes créées dans leur usage forceront les communautés et leurs responsables à aller dans une direction qui n'est pas forcément théologiquement assurée. Toute praxis chrétienne précède la theoria, encore faut-il voir en quoi une telle praxis est chrétienne.

Cet article se veut une tentative d'approche pastorale du phénomène virtuel en vue de l'accompagnement à la vie spirituelle ${ }^{2}$. La première partie se fonde sur l'expérience de la "gestion pastorale" de la crise liée à la pandémie vécue à Hong $\mathrm{Kong}^{3}$. Dans la seconde partie, nous allons procéder à une tentative d'analyse théologique du phénomène virtuel en nous appuyant d'abord sur l'enseignement du Magistère. Puis sur les travaux de Katherine Schmidt ${ }^{4}$ pour voir ce que recouvre le concept du réel appliqué à la communication à distance et comment cela participe à la communion. Et sur les travaux de Louis Marie Chauvet ${ }^{5}$ pour voir comment l'approche symbolique des sacrements décrite en termes de présent absent éclaire la communion en situation virtuelle. In fine pour savoir comment, dans cette communion, est présente la sacramentalité de l'Eglise. Cela nous permettra de voir quel type de communion il est possible de constater et comment cela pourra modifier le paysage pastoral futur en vue de l'accompagnement spirituel.

\footnotetext{
${ }^{2}$ Voir l'article: "Un besoin d'accompagnement spirituel après le confinement", La Croixmercredi 19 août 2020, Religion 13.

${ }^{3}$ Pour plus de détails on se référera à l'article de Rémy KuROwski, L'ecclésialité au défi du Covid-2019, l'expérience de Hong Kong, version électronique, site de Rémy Kurowski.

${ }^{4}$ Katherine G. Schmidt, Virtual Communion, Theology of the Internet and Catholic Imagination, Lexington Books/Fortress Academic, Lanham-Boulder-NewYork-London 2020. Dissertation Submitted to The College of Arts and Sciences of the University of Dayton, in Partial Fulfillment of the Requirements for The Degree of Doctor of Philosophy in Theology by Katherine G. Schmidt, Dayton, Ohio December 2016.

${ }^{5}$ Louis-Marie CHAUvET, "Symbole et sacrement, une relecture sacramentelle de l'existence chrétienne”, Paris, Cogitatio Fidei, no 144, Les Editions du CERF, juin 2008.
} 


\section{PARTIE: L'ECCLESIALITE AU DEFI DU COVID-19, L'EXPERIENCE DE HONG KONG}

\section{Contour général}

La pandémie en cours a surpris tout le monde, personne ne semblait préparé pour y faire face. L'ampleur du phénomène propagé dans l'espace-temps de la vie humaine sur terre ne laisse personne indifférent. Les restrictions imposées pour sauver les corps ont comme résonance dans les communautés chrétiennes (et certainement dans d'autres religions) la question de savoir comment sauver les âmes. Deux perspectives, deux manières différentes de réagir, mais le combat sur le terrain est commun. Côté spirituel, certains imaginaient même pouvoir arrêter la pandémie par des offrandes plus abondantes que d'ordinaire en augmentant aussi le régime de prière pour "chauffer à blanc" les centrales de purification des esprits et des corps pour lutter efficacement contre le couronnement de l'Antéchrist ${ }^{6}$. D'aucuns espéraient même des interventions divines spectaculaires. Est venu le temps du désert ${ }^{7}$. Une errance spirituelle et une impuissance scientifique se côtoient sans s'aimer vraiment. La puissance de la raison de la foi contre la puissance de la foi en la raison, les vieux tiroirs de croyances et de faits sont aérés ${ }^{8}$. D'aucuns pensent même pouvoir changer la place de certains tiroirs prouvant la supériorité de l'un sur l'autre. Dans l'ensemble, le scientisme pragmatique $^{9}$, avec des règles sanitaires imposant des gestes barrières, semble triompher de l'approche spirituelle. Les communautés scientifiques s'activent ébrouées par l'urgence, alors que les communautés spirituelles semblent, à l'instar des sociétés elles-mêmes tomber dans une sorte d'hébétude léthargique.

C'est dans ce contexte global que se déroule la pandémie à Hong Kong qui clôt (temporairement?) une longue et douloureuse marche de manifestations qui a secoué la RAS (Région d'Administration Spéciale) depuis de longs mois. La nouvelle loi sur la sécurité ${ }^{10}$ semble à l'avenir pouvoir peser aussi sur la vie

\footnotetext{
6 "A few days before Mr. Pelipenko set himself on fire, a bishop in the northern Russian region of Komi protested restrictions on churchgoing as an infringement of fundamental rights and threatened to go to court to get them reversed. Declaring that the ringing of church bells was the best way to combat the pandemic, he claimed that the word coronavirus - derived from the Latin word for «crown» - is «not coincidental but is linked to the coronation and enthronement of the Antichrist.»" The New York Times, 5 May 2020.

${ }^{7}$ La première phase de la pandémie s'est superposé avec le temps liturgique de Carême.

${ }^{8}$ Sur ce thème est consacrée une série de onze bandes dessinées, textes et dessins réalisés par Brunor (Bruno Rabourdin), Indices pensables, Brunor Editions, Paris 2009-2020.

${ }^{9}$ La gestion de la crise en Chine fondée sur le principe d'éradication totale contraste avec une approche plus souple adopte en Occident.

${ }^{10}$ En vigueur à partir du 1 juillet 2020.
} 
des communautés et de leur usage des moyens de communications. Forte de sa longue tradition de présence comme acteur majeur dans la construction de la société hongkongaise avec ses nombreuses institutions éducatives, caritatives et de santé, l'Eglise catholique de Hong Kong cherche aussi sa nouvelle place. Le diocèse de Hong Kong, en attente de nomination d'un nouvel évêque ordinaire $^{11}$, est depuis plus de 18 mois, sous la responsabilité d'un administrateur apostolique. Dans ce contexte, les indications sur la manière de célébrer ou de s'en abstenir tiennent lieu des orientations pastorales.

La CCFHK $^{12}$ est une aumônerie pour les francophones catholiques. La suspension des messes, du catéchisme et d'autres rassemblements est venue peu avant les vacances d'hiver ${ }^{13}$ occasionnant de nombreux départs prématurés. Très rapidement, la CCFHK s'est retrouvée vidée d'une part importante de sa substance en terme de personnes. Vite s'est posée la question de savoir comment les accompagner en sachant le danger de dispersion et d'isolement humain et spirituel. Danger renforcé par le fait d'une déferlante monothématique inégalée en termes de flux d'informations circulant par tous les canaux possibles. S'en défendre est une obligation sanitaire à un autre niveau : celui de la santé "intellectuelle", voire pour certains mentale, afin de garder intacte la capacité à analyser et maintenir l'équilibre, personnel et relationnel, parfois déjà si précaire, encore plus fragilisé par cette nouvelle situation. Comment alors non seulement s'en défendre du point de vue spirituel mais offrir aux autres dans l'entourage immédiat de quoi continuer à nourrir la leur dans cette nouvelle situation? Mens sana in corpore sano. A cet égard, dans la CCFHK, la prise de contacts individuels était parfois possible, restait la Newsletter par mail, mais cela s'est avéré peu efficace.

\section{Les nouveautés}

Elles se sont imposées d'elles-mêmes, par les circonstances qui les ont engendrées. La première fut celle venue comme réponse à l'absence de la messe. Une messe des Cendres transmise sur youtube pour ouvrir le carême, puis la mise en place d'un projet de rassemblement virtuel par zoom pour vivre ensemble la liturgie de la parole de Dieu, la seconde partie d'une telle "liturgie" étant envisagée à la maison comme geste symbolique à poser en lien avec

\footnotetext{
${ }^{11}$ Préaccord du 22 septembre 2018.

${ }^{12}$ Communauté Catholique Francophone de Hong Kong, le nom officiel donné à l'aumônerie. Avec à sa disposition un prêtre (l'auteur de cet article), un bureau, d'autres lieux (de cultes, de formations...) étant loués aux institutions ecclésiastiques ou pas. Elle est structurée comme une paroisse française avec une Équipe d'Animation Pastorale et un Conseil pastoral (et économique).

${ }^{13}$ Cette suspension fut levée en juin et ré-introduite 6 semaines plus tard.
} 
l'eucharistie dont on est privé ${ }^{14}$. Ce projet repose sur le schéma de l'église domestique vers où, dans cette nouvelle situation, se déplace le centre de gravité de la vie chrétienne; la vie communautaire étant symbolisée par la connexion virtuelle. Echo faible, peu de connexions, même en doublant la proposition samedi et dimanche. Il fallait renforcer les moyens de communiquer : whatsapp, facebook, instagram, en créant des groupes et plateformes d'échanges plus directes que ce que la traditionnelle Newsletter pouvait avoir comme effet. Pour maintenir le lien il a été décidé d'envoyer sur le groupe whatsapp de la CCFHK des méditations quotidiennes rédigées par l'aumônier comme fruit de sa prière du matin. Bon suivi et bons échos; pour beaucoup le seul moyen de nourrir leur prière et leur foi, et pour l'auteur se trouvant ainsi connecté une prise de conscience plus concrète de savoir pour qui, en plus de Dieu, il se lève tous les matins ${ }^{15}$.

Deux autres nouveautés sont venues en marge de la vie de la CCFHK, mais reliées à l'aumônier sollicité pour y prendre part. D'une part pour accompagner sur le plan théologique et conceptuel un groupe naissant (de prière, de formation et d'actions selon le schéma croire, vivre, célébrer) par zoom avec des catholiques du monde entier au même moment. D'autre part pour animer tous les dimanches une émission à La French Radio ${ }^{16}$ destinée aux francophones de Hong Kong et Macao, et d'ailleurs. Le premier, UPUM ${ }^{17}$, est en phase de création et nécessite un temps relativement long pour sa mise en place. Le concept de la radio est plus simple et plus facile à installer, mais les émissions (rédactions de textes et enregistrements) sont pour le moment assumées uniquement par l'auteur de cet article. Pour UPUM, la participation est fluctuante avec un noyau dur qui reste bien fidèle aux rendez-vous virtuels hebdomadaires (35-75 connexions parfois plusieurs personnes par connexion). Alors que l'audience de la radio est composée d'un groupe stable (plus de 300 connections) de personnes intéressées par les questions spirituelles et culturelles en lien avec la vie quotidienne et particulièrement dans cette période de pandémie. Dans les deux cas c'est un travail missionnaire qui est effectué, même si dans le premier les participants sont pour la

\footnotetext{
${ }^{14}$ Cf. le message de l'aumônier au Conseil pastoral, 25 février 2020, https//www.communautecathohk.com/

${ }^{15}$ Un message diffusé sur le whatsapp pour constater la déception devant si peu de connexions a été suivi d'un regain significatif depuis.

${ }^{16}$ Initialement la proposition a pour but de suppléer au manque de possibilité de rassemblement et d'enseignement religieux.

${ }^{17}$ United in Prayer, United in Mission, est une plateforme de rencontre pour tisser des liens ecclésiaux, offrir le soutien par la prière (du rosaire essentiellement), formation sur la nature propre de cette initiative (touchant à des domaines théologiques, pastoraux et de communication au travers le virtuel) et exprimer la fraternité (pouvant se prolonger en action caritative).
} 
plupart bien intégrés dans le tissu spirituel et souvent ecclésial local, ce qui ne semble pas être le cas majoritaire pour ceux de la radio.

\section{Questions que cela pose}

L'usage massif des moyens de communication à distance généralisé à la faveur de la pandémie et de ses restrictions dans les déplacements est pour l'Eglise autant une chance qu'un défi. La chance, plus facilement constatable que le défi, est une évidence : ce qui naguère était seulement réservé aux grandes entreprises, souvent internationales, s'est désormais démocratisé. Le Zoom par exemple en quelques semaines est sorti de son statut de luxe de la high tech pour devenir le 'prêt à porter' de la visioconférence appliquée à la vie de l'Eglise. Tout le monde s'est pris au jeu de la virtualité, chacun y trouvant son compte; les organisateurs en maintenant les contacts pour faire avancer les projets et leur suivi, les destinataires restant chez eux en gagnant du temps dans les déplacements. Par le biais du rapport à l'efficacité, le virtuel peut être un outil formidable favorisant la communion. Mais il peut aussi devenir un facteur supplémentaire de l'affaiblissement progressif du lien communautaire. Pendant ce temps-là, les fidèles cherchent avant tout de quoi nourrir leur vie spirituelle personnelle. Ils le font là où ils pensent pouvoir la trouver, les réseaux sociaux en regorgent, chacun se passe le mot pour en abreuver son entourage. Chacun capte où il peut ce qui lui semble être le meilleur pour nourrir surtout sa réflexion, mais souvent aussi sa vie spirituelle. Comment alors déplacer le curseur en donnant la priorité à la vie spirituelle incarnée?

Un autre aspect qui attire l'attention est celui de la valeur de l'information. Sur internet tout est à valeur égale, le besoin d'une herméneutique de sens par exemple concernant messes télévisées ou des bénédictions à distance se pose. Bien que le phénomène ne soit pas nouveau, l'Eglise n'échappe pas à cette déréliction latente qui se comprend aisément, tellement la pandémie a pris tout le monde de court. Le captage de communication tous azimuts semble avoir un caractère viral inflammatoire, engendrant une sorte de cacophonie rendant la parole de proximité difficilement audible. De toute évidence le choix de ce qui convient personnellement prime sur le choix à assumer communautairement. A l'occasion d'une telle profusion, on fait par exemple croître la connaissance de qui est le pape, de même pour un évêque ou un curé, en tant que personnes concrètes bien qu'enfermées dans leurs fonctions respectives, mais à qui, en cette occasion, va-t-on trouver un zeste supplémentaire d'humanité cachée jusqu'alors? Comment dans ce contexte se présente l'ecclésialité des rapports à l'intérieur de l’Église, cette réalité décrite en termes de Peuple de Dieu et de Corps du Christ ? 
L'ecclésialité à l'intérieur de laquelle les fidèles reçoivent de la nourriture spirituelle identifiée en terme de sacramentalité.

Les tamis d'Église se mettant progressivement en place existent, et leur efficacité est toute relative, tout en constituant déjà des indicateurs précieux d'une hiérarchisation possible ${ }^{18}$. C'est le premier travail auquel s'attelle tout responsable d'Église aux dimensions diocésaine et/ou paroissiale de savoir quoi proposer et pourquoi. En effet est en jeu la capacité d'inscription dans le tissu local du recours à de telles techniques. La vie de l'Eglise se réveille et se révèle aussi au moyen de tels outils et si ceux-ci ne servent pas à cela, l'ecclésialité perd de sa vigueur par les forces centrifuges qui poussent non pas à la décentralisation que l'on peut par ailleurs souhaiter, mais à la dispersion. L'accompagnement pastoral à la vie spirituelle se situe dans ce contexte.

Car tout l'enjeu est là : comment la pandémie actuelle qui produit, comme par effet de boomerang, une pratique nouvelle, révèle la vraie nature de la vie spirituelle des fidèles? L'individualisme latent qui sommeille dans tout être qui aspire à la liberté de pensée et d'action, est plus fort que l'invitation qui découle de la foi chrétienne à être un peuple qui se rend régulièrement visible comme Corps du Christ. De façon idéalisée spirituellement, il est plus facile d'imaginer l'appartenance au Corps du Christ sans pour autant en manger la présence. Alors qu'il est plus difficile d'envisager la réalité d'un tel Peuple de Dieu qui justement ne pouvant pas se rassembler, et par conséquent manger, peut cependant se contenter de rester dans la virtualité d'une telle relation au (corps du) Christ de la foi ${ }^{19}$. Et il est là ce danger redouté, soulevé, constaté par plusieurs dès le début de l'interdiction des rassemblements religieux. Dans ce contexte survivent les plus vaillants qui sans se laisser happer par la morosité ambiante ne s'arrêtent pas devant de telles difficultés. Si heureux sont ceux qui cherchent, comment le sont-ils ceux qui ne cherchent pas? Eux, cette majorité silencieuse des catholiques en temps normal de nom et de pratique sporadiques comme lien réel avec la communauté locale. La seule force d'attraction qui désormais s'exerce sur tous est celle qui les déracine, elle existait d'ordinaire mais elle était contrebalancée par ce lien naturel avec le lieu de culte. A l'intensité objective de l'attraction pour les déraciner du terreau local présente d'ordinaire s'ajoute donc l'interdit formel de tout rassemblement, l'interdit consenti par les responsables

\footnotetext{
${ }^{18}$ Hiérarchiser entre les informations reçues c'est aussi hiérarchiser entre deux catégories d'informations, celle qui concerne la communication par virtuel et le fait de maintenir la pratique d'aller à l'église, même si c'est sous une autre forme. On va donc insister par exemple sur le fait que les églises restent ouvertes et le chemin vers n'est pas barré, bien au contraire. Mais peu d'effet! Pour beaucoup l'interdit de messe publique est synonyme de la fermeture (physique) des portes de l'église.

${ }^{19}$ Une sorte de communion spirituelle qui remplace la communion eucharistique.
} 
d'Église à tous les niveaux. Passer sous silence les enjeux de la communion c'est laisser les fidèles en danger.

Dans le cas de la majorité silencieuse, la laïcisation teintée d'individualisme favorise d'abord la diminution, puis l'essai d'expulsion, et finalement d'exculturation $^{20}$ de la religion et du réflexe religieux dans le sens traditionnel du terme (prier tout seul et aller à la messe le dimanche), non seulement de la place publique, mais aussi purement et simplement de la mentalité ambiante. Dans ce contexte, le rappel du troisième commandement, celui de sanctifier le jour du Seigneur sonne creux, car résonne dans le vide cognitif à ce sujet, même parmi les plus attachés à la messe dominicale. La progression du phénomène de laïcisation se poursuit et il est à craindre qu'elle va, après avoir atteint des milieux traditionnellement déjà peu attachés à l'Eglise et ses rassemblements, toucher aussi de plus en plus fortement des familles encore attachées à l'Eglise catholique. Le décrochage se poursuit et, si on n'y prend garde, la pandémie risque de servir de catalyseur, un de plus. Si certains y resteront attachés, il est à craindre ${ }^{21}$ que pour une part d'entre eux, c'est plutôt dans un esprit protestant qu'ils vont poursuivre la route de la foi, car détachés du lien aux sacrements qui constituent la particularité catholique et qui ne sera plus décisive dans leur vie de foi.

Une telle vulnérabilité ne date pas d'aujourd'hui, mais dans ce contexte elle prend forme d'un avertissement plus sévère. L'instinct de survie, y compris de survie spirituelle, fait que l'on cherche une solution à tout prix et nombreux sont les effets bénéfiques ressentis comme tels par les intéressés eux-mêmes. Le pape François les qualifie d'emergency measures' ${ }^{22}$ qui ne peuvent pas remplacer les rassemblement liturgiques ordinaires. Il reste à savoir quelle est la valeur véritable de la virtualité du point de vue de l'ecclésialité, donc au bénéfice de la croissance du Corps du Christ, au bénéfice de l'approfondissement de leur foi? Seront-ils plus enracinés dans le tissu local lorsque la situation reviendra à la normale? Osons l'hypothèse que de tels bénéfices ne peuvent être envisagés que comme résultat de conversion du coeur à plus de foi et à plus de communion, qui s'ajoutera à la conversion à une meilleure posture dans le rapport au virtuel. Il est donc primordial d'envisager un accompagnement vers un tel ré-enracinement.

\footnotetext{
${ }^{20}$ Danièle Hervieu-Leger, Catholicisme, la fin d'un monde, Bayard, Paris, 2003. L'hypothèse de l'exculturation (terme original de l'auteur du livre) qui sous-tend l'ouvrage affirme que les justifications données à l'action de l'Eglise perdent progressivement toute consistance dans l'ensemble de la société française, même parmi les croyants pratiquants. Cf. chapitres II-IV, p. 55-167.

${ }^{21}$ Ceci est fondé sur les témoignages recueillis par l'auteur.

${ }^{22}$ Préface du livre de cardinal Walter KASPER, Diverse and United, I communicate therefore I am, The Vatican publishing House LEV 2020, où il note que la pandémie est un facteur provoquant le réveil spirituel.
} 
Celui-ci peut se faire au travers d'un projet pastoral délibérément mené dans ce sens dans lequel il s'agirait de mettre en lumière les bénéfices recueillis personnellement par chacun grâce aux liens virtuels de leur choix. Ces liens virtuels dans le cas du pape qui est reçu comme un super curé, peuvent être bénéfiques une fois que les bénéficiaires vont se laisser accompagner vers une meilleure compréhension des enjeux de l'ecclésialité au travers la communion à vivre dans le cadre de la communauté locale, ce à quoi le pape actuel ne cesse de renvoyer ${ }^{23}$.

L'enjeu de l'ecclésialité se situe au niveau de l'efficacité, c'est l'efficacité qui éprouve l'ecclésialité. L'efficacité au sens purement technique du terme cherche à obtenir le meilleur résultat dans les meilleures conditions pour parvenir au but recherché, à savoir au moindre coût et dans les plus brefs délais ${ }^{24}$. Alors que l'ecclésialité repose avant tout sur le principe de l'efficience ${ }^{25}$ qui est une prise en compte de l'ensemble des relations à considérer comme une capacité grâce à laquelle les résultats d'ordre spirituel sont tôt ou tard obtenus. C'est la qualité des relations qui est l'enjeu majeur se trouvant au centre de l'ecclésialité. La vie spirituelle en dépend. Son irrigation par les moyens virtuels porteurs eux aussi de sacramentalité peut renforcer l'ecclésialité. Dans ce contexte, comment la communication virtuelle favorise la communion, en plus ou en moins, suivant les circonstances, c'est ce qui reste à faire pour fonder théologiquement l'accompagnement pastoral (et donc spirituel) de communautés et de leurs membres.

\footnotetext{
23 "Il y a une tension entre la globalisation et la localisation: « Il faut prêter attention à la dimension globale pour ne pas tomber dans une mesquinerie quotidienne. En même temps, il ne faut pas perdre de vue ce qui est local, ce qui nous fait marcher les pieds sur terre. L'union des deux empêche de tomber dans l'un de ces deux extrêmes ». (EG, n. 234) Le disciple missionnaire n'est pas obsédé par des questions limitées et particulières: il élargit son regard pour reconnaître un bien plus grand bénéfique à tous. Il respire aux dimensions de l'Église (géographiquement) universelle. Cependant il est nécessaire qu'il enfonce ses racines dans l'histoire et la terre de son propre lieu. Il travaille sur ce qui est petit, mais dans une perspective plus large; il ne renie pas son identité mais s'intègre cordialement dans une communauté plus large. Il ne se laisse tromper ni par la sphère globale qui annihile, ni par la partialité isolée qui rend stérile. (EG, n. 235)". La joie de l'Evangile, 2013.

24 "Je préfère de regarder la messe par zoom cela me fait gagner du temps" avoue tout simplement une adolescente par ailleur bien éveillé à la foi et la spiritualité.

${ }^{25}$ Le terme efficience, un néologisme, est employé dans le sens donné par l'Académie Française: "capacité d'agir en produisant un effet attendu" s'appliquant aussi à un réseau de communication, alors que le terme efficacité est employé dans le sens d'une réalisation de l'objectif le plus rapidement et le plus simplement, au moindre coût possible.
} 


\section{PARTIE: ESSAI D’UNE ANALYSE THÉOLOGIQUE DU VIRTUEL}

Pour analyser théologiquement le phénomène du virtuel comme moyen de communication dans l'Eglise, je m'appuie sur la thèse de Katherine Schmidt. En conclusion de son livre l'auteur constate que "[...] internet has become a potential site for communion." ${ }^{, 26}$ Elle étudie tous les moyens de communications disponibles, mais applique cette conclusion en particulier à l'eucharistie. Les messes télévisées sont donc visées pour savoir si une telle mise à distance provoque non pas, oui ou non, mais comment, une modification dans la communion qui non seulement est l'objectif de toute communication, mais aussi dans l'Eglise et pour la foi chrétienne, constitue le point central de son identité et point final de son action.

Elle appuie sa conclusion positive sur le constat de la possibilité d'identifier une telle potentialité au moyen d'une approche symbolique telle qu'elle est exposée par L-M Chauvet. Approche dans laquelle le concept absent présent permet de prendre en compte la gratuité de l'amour de Dieu qui se laisse découvrir dans l'Eucharistie. L'approche symbolique des sacrements et de la sacramentalité dans son ensemble, où le concept présent absent est appliqué peut être utilisé pour comprendre le lien avec la réalité du virtuel. Ceci est considéré par Schmidt comme une nouvelle porte d'entrée pour le comprendre théologiquement comme partie prenante de la vie de l'Eglise ${ }^{27}$. L'approche symbolique est un concept dans lequel une telle sacramentalité se reflète et prend sa part active. Il devient un creuset pour la réflexion théologique sur sa pertinence dans l'agir chrétien. Pour Schmidt, en reconnaissant un tel potentiel de communion dans l'Eglise, il est possible d'élargir l'espace d'action de l'Église ${ }^{28}$ ainsi équipée et d'explorer dans l'esprit missionnaire les points de contacts avec le monde. Il est aussi applicable à l'accompagnement pastoral.

Le virtuel est une chance pour l'Eglise qui élargit ainsi l'espace cognitif de son action ad extra tout en l'intensifiant ad intra. C'est "l'endroit" où le fidèle peut s'épanouir dans sa foi et où le monde peut rencontrer l'Eglise. Tout en sachant que les médias qui favorisent l'immédiateté montrent en même temps l'impossibilité de remplir ce désir d'un réel tel que celui-ci est espéré, dans sa corporéité même. Entre l'immédiateté et la corporéité il y a une distance que le virtuel impose et dont la théologie dispose. L'absence se montre plus tenace que

\footnotetext{
${ }^{26} \mathrm{~K}$. SCHMidT, Virtual communion, Theology of the Internet and the Catholic Sacramental Imagination, p. 155.

27 "Chauvet`s theological system provides a framework for understanding internet" "Le concept théologique de Chauvet fournit la base pour la compréhension de l'internet", in K. SCHMIDT, p.123.

28 "[...] Christian liturgy can be understood as virtual. This is an expansion and concretization of the idea of religion as mediatio", in K. SCHMIDT, p. XII.
} 
ce que le désir d'immédiateté pouvait suggérer comme constat de présence. En tenir compte c'est avancer dans une exploration sans se résigner à des jugements de validité trop précoces ou ceux de non validité hâtive.

Son travail couvre la totalité des moyens de communication par internet dans tout ce que la technologie moderne permet actuellement. Elle dénombre cinq domaines dont la théologie doit se saisir : anonymat, critique amère, autorité, accessibilité et désincarnation ${ }^{29}$. Même si tous sont connectés entre eux, c'est le dernier qui est au centre de notre présentation. Le virtuel pose avant tout la question de la présence de la corporéité, qui est à regarder du point de vue du principe d'incarnation et de ce qu'est l'Eglise en tant que corps du Christ. Dans cette perspective nous allons examiner ce que recouvre le réel et le non réel, puis comment cela se traduit en terme d'immédiateté et de médiation, et comment cela influe sur le rapport entre l'eucharistie et la charité. Mais avant, une brève présentation du positionnement du magistère face l'émergence de ces moyens de communication.

\section{Les Médias et l'Église}

Dans la première phase, dès leur apparition, comme nous l'avons déjà mentionné dans l'introduction générale, les médias sont d'abord considérés comme un moyen de communication pour l'Eglise ad extra, dans l'esprit missionnaire. L'Eglise, en observatrice attentive de l'émergence des nouveautés technologiques, veillait surtout à ce que la morale qui y était véhiculée soit bien conforme avec l'enseignement de l'Eglise. Le cinéma et la radio sont considérés comme des véhicules de la culture catholique de la vertu ${ }^{30}$ et de la promotion de la nature de l'homme à l'image de Dieu. Pie XII entrevoit aussi le rôle que peuvent jouer les médias un rôle dans l'évangélisation ${ }^{31}$. Demeure le regard suspicieux à l'égard des médias, considérés comme un produit artificiel, étranger à l'espace chrétien.

Le concile Vatican II va opérer un premier changement, en y identifiant leur aptitude à être au service des communications sociales qui participent au bien commun en vue du Royaume de Dieu ${ }^{32}$. Jean Paul II y verra la possibilité

${ }^{29}$ K. SCHMIDT, chapitre I, pages 1-24.

${ }^{30}$ PIE XI, Divini illius Magistri, qui présente la figure du Christ bon pasteur et docteur, in K. SCHMiDT, p. 28.

${ }^{31}$ PIE XII, Miranda Prorsus, in K. SchmidT, p. 30.

${ }^{32}$ Concile Vatican II, Inter Mirifica, Décret sur les moyens de communication sociale, no 2: "Ils apportent une importante contribution...à l'extension et à l'affermissement du Royaume de Dieu", tout en exprimant "un souci vigilant". 
donnée à la liberté humaine de s'exprimer. ${ }^{33}$ Le second changement s'opère par Benoît XVI qui attire l'attention sur le danger de la distorsion du réel qui s'opère dans les communications sociales. "Today, communication seems increasingly to claim not simply to represent reality, but to determine it, owing to the power and the force of suggestion that it possesses"34. Le pape pose la question de la communion des êtres charnels et ceci en vertu de l'incarnation de l'Evangile dans un monde réel, en lien avec les visages réels de nos frères et soeurs avec qui nous partageons notre vie (idem).

En les intégrant à l'intérieur de son espace ecclésial, l'Église peut s'enrichir en considérant les moyens de communication comme une part de l'histoire humaine et de la culture, et même comme médiateurs des choses spirituelles. ${ }^{35} \mathrm{Si}$ les autorités ecclésiastiques ont à consulter les experts des médias, ce n'est pas pour leur laisser la main pour savoir comment les utiliser dans l'Église. Ceci est à la charge de l'Eglise où s'élabore l'intégration théologique dans le tissu ecclésial en vue de la sacramentalité $e^{36}$, agent actif de la communion. On les voit comme service rendu aux personnes isolées ${ }^{37}$, comme possibilité d'annonce de l'Evangile (Benoît XVI), ou encore comme possibilité de rencontre et de solidarité (pape François). Dernièrement, dans Laudato si, le pape François attire l'attention sur la pollution mentale. Tous les papes situent les médias dans la perspective de communion.

\section{Réel et non réel}

Qu'est-ce qui est vrai dans le virtuel? Deux positions ontologiques antagonistes se côtoient. L'une réaliste, objective et causale, l'autre nominaliste et subjective où le réel est réellement existant dans la compréhension personnelle et son interprétation du monde physique. Comment, dans un cyberespace, la corporéité persiste-t-elle? Au travers de son identité et donc de ce qu'elle désire y mettre, constate une étude sur l'identité de la communauté virtuelle ${ }^{38}$.

Ce qui plaide en faveur de l'hypothèse nominaliste qui subjective le rapport au réel, c'est que pour une part ce rapport est objectivable. La question qui se pose alors, c'est de savoir si une telle communication visant la communion est

\footnotetext{
${ }^{33}$ JEAN PAUl II, in K. SCHMIDT, p. 43-46.

${ }^{34}$ BenOIT XVI, Message pour la journée de médias et communication 2008, in K. SCHMIDT, p. 47.

${ }^{35}$ PIE XII, Miranda Prorsus, in K. SCHMIDT, p. 31.

${ }^{36}$ Aetatis Novae, 1992, guide pour la meilleure intégration des communications sociales dans la paroisse et dans le diocèse, in K. SchmiDT, p. 38.

${ }^{37}$ Ethique de l'Internet, Conseil Pontifical pour les communications sociales, 7, in K. SCHMIDT, p. 61.

${ }^{38}$ Judith S. DONATH, Identity and Deception in the virtual community, 1996, Massachusetts Institute of Technology, Cambridge, MA, United States.
} 
limitée ou, au contraire améliorée. Sur le plan formel on répondra limitée, mais sur le plan subjectif, améliorée. Elle est à la fois limitée et améliorée, ce qu'illustre l'exemple (que l'on pourrait considérer comme fallacieux par ailleurs) de l'attitude où on met en avant le gain de temps et la limitation de fatigue liée aux déplacements. On ne peut pas non plus considérer la question des moyens de communications à distance en termes opposés, réel-virtuel, ce dernier classifié comme non réel. Le terme virtuel comporte le sens d'une proximité avec le réel, mais il n'est pas totalement réel ${ }^{39}$. Le corps peut être vu mais pas touché. Un tel constat oblige à prendre en compte une nouvelle manière de considérer l'espace comme lieu du réel, espace plus diffus, moins corporel, plus expérimental, moins identifiable ${ }^{40}$. Le rêve d'une totalement "réalistique" réalité virtuelle (Star Trek), peut trouver son antidote dans les communautés locales de l'Eglise ${ }^{41}$.

\section{Présent absent ${ }^{42}$}

L'approche symbolique proposé par Chauvet s'appuie sur le réel tout en dépassant les cadres ontologiques que la métaphysique pouvait fournir. Fort des apports fournis par les sciences modernes qui intègrent dans le réel de l'existence humaine, le langage, les sentiments... Il y a deux sortes d'échanges : mercantiles, procédant selon le principe du donnant-donnant où sont échangés des objets à valeur estimée; et les échanges de dons qui, dans un jeu de gratuité de l'échange, impliquent l'engagement personnel (oblatif) total. L'échange symbolique est la forme "liturgique" d'une telle circulation de dons. Dans l'échange symbolique sont échangées l'identité du sujet et sa place dans la société, donc dans la communauté. Cet échange symbolique non pas moins réel implique la présence absence par leur non saturation respective. Dans cette perspective, la virtualité devient le lieu de l'herméneutique de la sacramentalité de l'Eglise et de sa pertinence dans l'espace humain ${ }^{43}$.

$\mathrm{Du}$ point de vue théologique on peut se poser la question de savoir comment la grâce soutient les deux. Comment la grâce devient ce troisième

\footnotetext{
${ }^{39}$ Jaron LANIER, "Virtual as a sensory-motor mirror opposite of the human body", in. K. SCHMIDT, p. 60 .

${ }^{40}$ Graham WARD, "Between virtue and Virtuality", Theology Today 59 no. 1 (2002), in K. SCHMIDT, p. 17.

${ }^{41}$ Jean PaUl II, Rights and Duties of the Recipients in Social Communication 1978, in K. SCHMIDT, p. 18.

${ }^{42}$ Rainer Maria Rilke (1875-1926), le poète autrichien est auteur de l'expression absence ardente, cf. Lucques ClAIRE, L'absence ardente, visages de Rilke, La Renaissance, 1977.

${ }^{43}$ Le troisième chapitre du livre de Schmidt y est entièrement consacré.
} 
élément entre le présent et l'absent et comment elle se manifeste au coeur même du corps humain et dans l'image de celui-ci lorsqu'il est virtuellement présent aux autres? L'absence du corps du Christ dans l'Eglise primitive prend immédiatement la forme d'une médiation sacramentelle entre la présence et l'absence ${ }^{44}$. Dans cette même logique sont accomplis les sacrements et toute la sacramentalité qui en découle. Cette médiation sacramentelle s'exprime dans les pèlerinages virtuels, (comme le chemin de croix, voire le rosaire). C'est vrai pour les lettres pauliniennes, c'est vrai pour la notion du corps mystique de l'Église...Tout ceci d'une manière ou d'une autre participe à la dialectique présence absence. L'échange symbolique fondé sur l'offrande des personnes d'elles-mêmes, produit une tension entre présence et absence que l'on pourrait définir en termes de virtuel ${ }^{45}$. En découle l'obligation d'accepter le caractère inachevé et imparfait d'une telle situation.

\section{Immédiateté et médiation}

Nous venons de le mentionner, la médiation sacramentelle interpelle le rapport au temps. C'est la peur de l'absence qui pousse à l'immédiatetée ${ }^{46}$. "N'ayez pas peur" face à toutes les tempêtes qui finissent par être apaisées, c'est accepter l'absence de la réelle communion qui suppose immédiateté de présence et de ses effets. C'est le cas de l'absence de manducation, manger l'hostie, lors de la messe télévisée. Dans les situations des personnes incapables de se rendre à l'église c'est l'Église qui vient en la personne des diacres qui portent la communion, (cf. les services évangéliques de malades). Accepter une telle absence se pose alors en termes d'exigence, et non seulement de substitution ordinaire, même dans l'approche de la communion spirituelle ${ }^{47}$.

Même si toute médiation n'est pas subordonnée au bien, le désir d'immédiateté, si fort dans le virtuel, affecte l'approche des sacrements. Le virtuel s'impose avec sa dialectique propre comme une remédiation supplémentaire. La logique virtuelle est au centre de l'espace cognitif imaginaire de l'Eglise. L'hyper médiation et la transparence sont la manifestation d'un désir contradictoire de dépasser les limites de la représentation et d'atteindre le réel. Dans la perspective de l'incarnation cette logique est essentielle pour l'imaginaire chrétien. Elle

${ }^{44}$ De Certeau, in K. SCHMIDT, p. 67-68.

${ }^{45}$ Louis-Marie Chauvet consacre chap.III à la médiation, in K. SCHMIDT, p. 140.

${ }^{46}$ Louis-Marie Chauvet, in K. SCHMIDT, p. 137.

${ }^{47} \mathrm{La}$ communion spirituelle est un concept catholique selon lequel la communion physique au Corps du Christ lors de la messe ou en dehors, dans les cas d'empêchements peut être remplacée par une communion du désir, cf. Aleteia, Communion spirituelle, de quoi parle-t-on? 10 avril 2020, fr.aleteia, org. 
donne du corps au désir humain de se laisser emporter par l'hyper médiation au travers de l'intimité avec le Dieu Créateur. Pour le chrétien, le désir d'immédiateté se traduit par le désir de communion avec Dieu. L'hyper médiateté et l'immédiateté se trouvent paradoxalement réconciliées dans le désir de communion eucharistique. Encore faut-il pouvoir y accéder.

\section{Communion et charité}

Comment le virtuel, change-t-il, défie-t-il et affecte-t-il la vie de la communauté? Il incite à regarder de plus près la relation entre la liturgie et l'éthique. La liturgie, à l'instar du Christ, est un puissant pédagogue qui apprend comment accepter l'absence de Dieu, ce qui oblige à lui donner notre corps dans ce monde. Comment dans le virtuel s'effectue le sacrement comme liturgie du prochain? Ce qui est échangé dans la liturgie eucharistique c'est, à l'image du Christ luimême, la vie même de ses membres.

La faiblesse de conscience d'ordinaire d'une telle implication dans la messe dans une assemblée, se reporte sur la situation de la messe 'suivie' de façon virtuelle. Cette faiblesse nourrit l'interprétation de ce qu'est la messe : non seulement elle demeure tout aussi superficielle, mais en plus, le virtuel aggrave la situation car il provoque l'éloignement de la confrontation réelle et immédiate avec le prochain. Sans pour autant exclure de pouvoir nourrir quelques espoirs pour une telle confrontation plus tard, mais de façon nouvelle, plus individuelle, voire assumée, mais plus facilement déconnectée de la vie de la communauté qui célèbre.

L'expérience de l'utilisation du virtuel dans le travail pastoral à Hong Kong durant la période de pandémie démontre la nécessité d'élaborer des outils d'analyses avec une grille de lecture adaptée. Une lacune qu'il est urgent d'essayer de combler.

\section{BIBLIOGRAPHIE}

Aetatis Novae, instruction pastorale du Conseil pontifical pour les communications sociales; guide pour la meilleure intégration des communications sociales dans la paroisse et dans le diocèse, Vatican, 17 mars 1992.

BENOIT XVI, Message pour la journée de médias et communication, Vatican 2008.

BRUNOR (BRUNo RABOURDIN), Indices pensables, série de onze bandes dessinées, Brunor Editions, Paris 2009-2020.

CHAUvET, Louis-Marie, Symbole et sacrement, une relecture sacramentelle de l'existence chrétienne, Cogitatio Fidei, no. 144, Les Editions du CERF, Paris 2008.

Claire, Lucques, L'absence ardente, visages de Rilke, La Renaissance, 1977. 
Concile Vatican II, Décret sur les moyens de communication sociale, 4 décembre 1963.

Donath, Judith S., Identity and Deception in the Virtual Community, Massachusetts Institute of Technology, Cambridge, MA United States 1996.

Ethique de l'Internet, Conseil Pontifical pour les communications sociales, 28 février 2002.

FrançoIs, La joie de l'Evangile (EG), exhortation apostolique, Mame Editions 2013.

Hervieu-Leger, Danièle, Catholicisme, la fin d'un monde, Bayard, Paris 2003.

KASPER, Walter, Diverse and United, I communicate therefore I am, The Vatican publishing House LEV 2020.

KuRowski, Rémy, "Internet, Lieu d'Église virtuel”, in : Cahier de l'Atelier $\mathrm{n}^{\circ}$ 549, avril-juin 2011, et La Documentation catholique $\mathrm{n}^{\circ} 2475,2$ octobre 2011, p. 834-838.

KuRowski, Rémy, L'ecclésialité au défi du Covid-2019, l'expérience de Hong Kong, version électronique site de Rémy Kurowski.

La Croix-mercredi 19 août 2020, Religion 13.

Schmidt, Katherine G., Virtual Communion, Theology of the Internet and Catholic Imagination. Lexington Books/Fortress Academic, Lanham-Boulder-NewYork-London 2020. Dissertation Submitted to The College of Arts and Sciences of the University of Dayton, in Partial Fulfillment of the Requirements for The Degree of Doctor of Philosophy in Theology by Katherine G. Schmidt, Dayton, Ohio December 2016.

PIE XI, Divini illius Magistri, encyclique, 31 décembre 1939.

The New York Times, 5 May 2020.

WARD, Graham, "Between virtue and Virtuality", Theology Today 59 no. 1(2002).

\section{WIRTUALNOŚĆ I SAKRAMENTALNOŚĆ W ŻYCIU DUCHOWYM}

\section{STRESZCZENIE}

Obecna pandemia zwiększa wykorzystanie zdalnych środków komunikacji. To, co wirtualne, już bardzo obecne w duszpasterskiej tkance Kościoła, nabiera intensywności. Stawia też pytania chrześcijańskiej teologii. Doświadczenie Hongkongu pozwala postawić pytania i zasugerować pewne kierunki myślenia dla działalności duszpasterskiej. Wykorzystanie środków wirtualnych rodzi pytania o skuteczność, konieczność ustalenia priorytetów propozycji, zobowiązuje do ponownego przemyślenia stosunków kościelnych, ujawnia ukryty indywidualizm oraz kruchość więzi z Ciałem Chrystusa i przynależności do Ludu Bożego, uczy instynktu duchowego przetrwania z jednej strony i wrażliwości z drugiej. Aby uwzględnić taką nowość, w centrum odpowiedzi znajduje się propozycja nawrócenia serc, której towarzyszą szczegóły dotyczące teologicznej wartości prawdziwej komunii poprzez tzw. komunikację wirtualną.

Słowa kluczowe: komunikacja; komunia realna; komunia wirtualna; eklezjalność; sakramentalność; duchowość; duszpasterstwo. 